\title{
An Unusual Cause of Protein Losing Enteropathy in a 2.5-Year-Old Girl: Meso-Intestinal Fibrosis
}

\author{
Fatemeh Mahjoub ${ }^{1} \quad$ Mehri Najafi Sani ${ }^{2} \quad$ Ahmad Khaleghnejad Tabari ${ }^{3} \quad$ Maryam Monajemzadeh $^{4}$ \\ Saeed Zandieh ${ }^{4}$
}

${ }^{1}$ Department of Pathology, Valiasr Hospital, Imam Khomeini Hospital Complex, Tehran University of Medical Sciences, Tehran, Islamic Republic of Iran

2 Department of Gastroenterology, Tehran University of Medical Sciences, Tehran, Islamic Republic of Iran

${ }^{3}$ Department of Pediatric Surgery, Shahid Beheshti University of Medical Sciences, Tehran, Islamic Republic of Iran

${ }^{4}$ Department of Pathology, Tehran University of Medical Sciences, Tehran, Islamic Republic of Iran
Address for correspondence Fatemeh Mahjoub, Department of Pathology, Valiasr Hospital, Imam Khomeini Hospital Complex, End of Keshavarz Boulevard, Tehran 14194, Islamic Republic of Iran (e-mail: fmahjoub@sina.tums.ac.ir).

Eur J Pediatr Surg Rep 2014;2:29-31.

\begin{abstract}
Keywords

- meso-intestinal fibrosis

- protein losing enteropathy

- pediatric age group

Introduction Protein losing enteropathy is a symptom characterized by loss of protein in intestines resulting in low protein levels in serum and generalized edema. Several causes are reported for this condition. Hereby we report an as yet unreported cause of protein losing enteropathy that we named meso-intestinal fibrosis.

Case Report A 2.5-year-old girl referred with features of partial intestinal obstruction and underwent laparotomy. She had history of protein losing enteropathy since 16 months of age with generalized edema and received albumin every other week. Workup of protein losing enteropathy was inconclusive and only a histology report denoted increase in eosinophils in lamina propria of small intestine and hypoallergenic diet was started for her, but no significant response was noted. Laparotomy revealed lace-like white areas in meso of small intestine and intestinal wall was firm in palpation in some areas. Biopsy was taken from these sites and histology revealed severe fibrosis of meso overlying muscularis propria and also patchy fibrosis of intestinal meso led to severe lymphangiectasis in submucosa of small intestine.

Discussion Secondary lymphangiectasis due to obstruction of lymphatic flow is mentioned as cause of protein losing enteropathy. Meso-intestinal fibrosis seen in this case that led to secondary lymphangiectasis and also motility disorder has not been reported as yet.
\end{abstract}

\section{Introduction}

Protein losing enteropathy (PLE) is a rare condition characterized by increased excretion of protein in feces leading to hypoproteinemia and generalized edema with ascites and plural and pericardial effusions. ${ }^{1}$ PLE is a diagnosis of exclu- sion (after ruling out proteinuria, malnutrition, and impaired protein synthesis due to hepatic cirrhosis). After exclusion of other causes, the most reliable method to determine enteric protein loss is to assess the clearance of $\alpha_{1}$-antitrypsin from plasma. ${ }^{1}$ Several causes have been described for this condition such as cardiac conditions, ${ }^{2}$ lymphatic obstruction due to received

February 10, 2013

accepted after revision

May 6, 2013

published online

May 31, 2013
DOI http://dx.doi.org/ 10.1055/s-0033-1348040. ISSN 2194-7619. (c) 2014 Georg Thieme Verlag KG
Stuttgart · New York

License terms

(ㄷ) (1) $\ominus$ (2) 
malignancy (lymphoma), and gastrointestinal causes. ${ }^{1}$ Gastrointestinal causes of PLE are either erosive or nonerosive. The most common etiology of nonerosive PLE is Menetrier disease, which is rare in pediatric age group and is characterized by hypertrophic gastric folds. ${ }^{3}$ Other nonerosive gastrointestinal conditions that may be complicated by PLE include eosinophilic gastroenteritis, small intestinal bacterial overgrowth, celiac disease, microscopic colitis, and Helicobacter pylori infection. ${ }^{1}$ Ulcerative colitis and Crohn disease are among erosive gastrointestinal diseases leading to PLE. ${ }^{4}$

In pediatric age group, primary lymphangiectasis is seen rarely, which is characterized by the presence of dilated lymphatic channels in tips of small intestinal villi. ${ }^{5}$ Secondary lymphangiectasis is seen mainly due to lymphatic obstructions. ${ }^{1}$ A recently published article denoted an infant with rotavirus infection who presented with PLE. ${ }^{6}$

Congenital malformations of lymphatics and retroperitoneal fibrosis are stated briefly as causes of PLE. ${ }^{1}$

Hereby we intend to report a case of unusual PLE due to meso-intestinal fibrosis that led to secondary lymphangiectasis which we believe is yet an unreported entity.

\section{Case Report}

A 2.5-year-old girl referred with features of partial intestinal obstruction and underwent laparotomy. She had history of PLE since 16 months of age with generalized edema and received albumin every other week. Workup of PLE was inconclusive and only a histologic report from duodenal biopsy taken during upper endoscopy noted an increase in eosinophils in lamina propria of small intestine. Hypoallergenic diet was started for her, but no significant response was noted. She also had a history of intermittent vomiting since 5 months of age treated with motilin and ranitidine with diagnosis of gastroesophageal reflux disease. Vomiting increased in frequency from once a month to twice a week in recent months and each episode ended in bile vomiting. Partial obstruction was suggested for her and workup for intestinal obstruction was performed. Plain abdominal radiography before laparotomy revealed local obstruction with dilation of small intestinal loops. Spiral abdominal computed tomography scan revealed an increased circumferential wall thickness of segments of jejunum with obstruction of loops proximal to it, suggesting infiltrative lesions. Laparotomy revealed no visible obstruction but lace-like white areas in meso of small intestine. Intestinal wall was firm in palpation in some areas. Biopsy was taken from these sites and histology revealed normal mucosal villi ( - Fig. 1A), the presence of dilated lymphatic channels in submucosa (-Fig. 1B), severe fibrosis of meso overlying muscularis propria (-Fig. 1C), and also patchy fibrosis of intestinal meso ( $-\mathrm{Fig}$. 1D) led to severe lymphangiectasis in submucosa of small intestine. After laparotomy, the vomiting episodes are stopped, but she receives albumin to control hypoproteinemia as before. However, with institution of Caprilon (Nutricia Ltd., Wiltshire, UK) (having medium chain fatty acids), the need for albumin infusion is reduced.
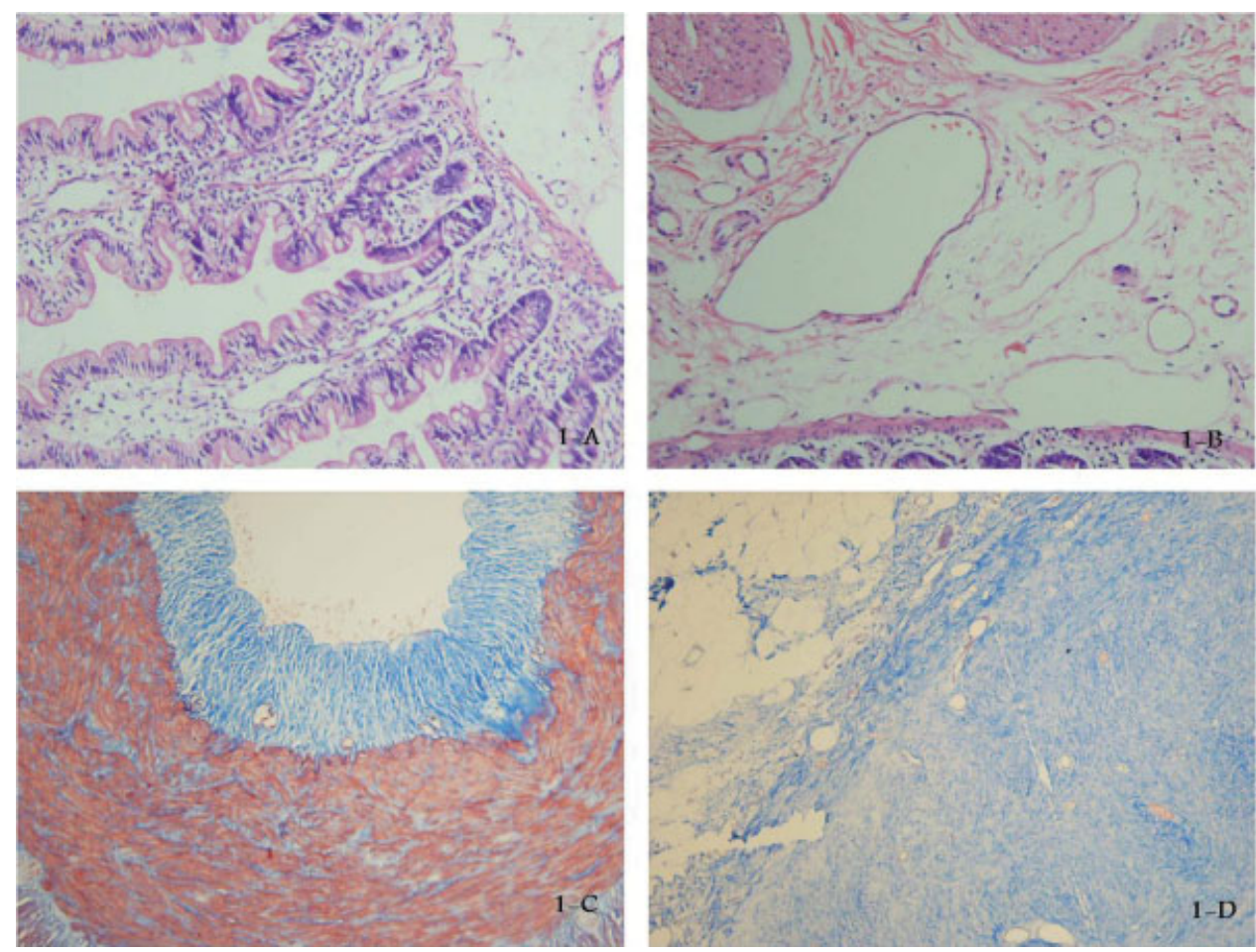

Fig. 1 (A) Small intestinal mucosa with rather tall villi and no significant inflammatory infiltrate; hematoxylin and eosin stain $(\times 40)$. (B) Small intestinal submucosal with the presence of several dilated lymphatic channels; hematoxylin and eosin stain $(\times 40)$. (C) Small intestinal wall with severe fibrosis of meso overlying muscularis propria (blue colored area overlying muscularis propria); trichrome stain ( $\times 40)$. (D) Small intestinal meso with severe patchy fibrosis. There is no inflammatory infiltrate in fibrotic areas and other sites as well; trichrome stain $(\times 40)$. 


\section{Discussion}

PLE is mainly a diagnosis by exclusion. It occurs rarely in pediatric age group. Most frequent causes of this condition are cardiac and gastrointestinal disorders. Gastrointestinal causes are divided into nonerosive and erosive conditions. ${ }^{1}$ We investigated rather all the above-mentioned causes in our case and no stated hallmarks of these disorders were found. Because of partial obstruction symptoms, laparotomy was performed and unusual findings such as severe fibrosis of meso overlying muscularis propria of the small intestine were observed. No such case was encountered in a thorough research of literature using the keyword of intestinal fibrosis. Articles found with the keyword of intestinal fibrosis or mesointestinal fibrosis were mostly dedicated to localized fibromatosis of intestine and patients were not referred with picture of $\mathrm{PLE}^{7,8}$ The most relevant condition is sclerosing mesenteritis, which is rarely seen in pediatric age group. In a case report concerning a 6-year-old girl, presenting features were growth failure and chronic anemia ${ }^{9}$ rather than PLE. The aforementioned case also shows evidences of inflammatory process in the intestinal wall, which is not observed in our case.

Another complication of fibrosis of the intestinal wall appears to be impaired motility with signs and symptoms of partial intestinal obstruction in this case.

Parents are close relatives (cousins) and she is the only child of the family. Although family history is negative for an identical case, the possibility of genetic disorders should be kept in mind.

In conclusion, our case appears to be a newly encountered cause of PLE and the prognosis and treatment options are not known for this patient.

\section{Acknowledgments}

Hereby we thank Professor Elizabeth Montgomery (John's Hopkins University, Baltimore, MD, United States) for reviewing the case and Dr. Reza Mahjoub for editing the text. We also thank Ms. Besharati and Ms. Omidi for their kind technical support.

\section{Conflict of Interest}

None

\section{References}

1 Umar SB, DiBaise JK. Protein-losing enteropathy: case illustrations and clinical review. Am J Gastroenterol 2010;105(1):43-49, quiz 50

2 Davidson JD, Waldmann TA, Goodman DS, Gordon RS Jr. Proteinlosing gastroenteropathy in congestive heart-failure. Lancet 1961; 1(7183):899-902

3 Toubia N, Schubert ML. Menetrier's disease. Curr Treat Options Gastroenterol 2008;11(2):103-108

4 Ferrante M, Penninckx F, De Hertogh G, et al. Protein-losing enteropathy in Crohn's disease. Acta Gastroenterol Belg 2006;69 (4):384-389

5 Roberts JD, Goldstein AM. Gastrointestinal tract and pancreas. In: Gilbert-Barness E, ed. Potter's Pathology of the Fetus, Infant and Child. 2nd ed. St. Louis, MO: Mosby; 2007:1198

6 Iwasa T, Matsubayashi N. Protein-loosing enteropathy associated with rotavirus infection in an infant. World J Gastroenterol 2008;14(10):1630-1632

7 Polat C, Aktepe F, Turel S, Yazicioglu B, Ozkececi T, Arikan Y. A giant mesenteric fibromatosis case presenting with mechanical intestinal obstruction and successfully resected with partial duodenojejunectomy and right hemicolectomy. Clinics (Sao Paulo) 2010;65 (1):110-113

8 Wronski M, Ziarkiewicz-Wroblewska B, Slodkowski M, Cebulski W, Gornicka B, Krasnodebski IW. Mesenteric fibromatosis with intestinal involvement mimicking a gastrointestinal stromal tumour. Radiol Oncol 2011;45(1):59-63

9 Viswanathan V, Murray KJ. Idiopathic sclerosing mesenteritis in paediatrics: report of a successfully treated case and a review of literature. Pediatr Rheumatol Online J 2010;8:5 